\title{
Unravelling the network
}

White-opaque switching in Candida albicans occurs stochastically at low frequency and is heritable for many generations. White and opaque cells are genetically identical, yet these cells show profound differences in morphology, virulence properties and gene expression. The detailed molecular mechanism that is responsible for this heritable epigenetic switch has now been revealed by Rebecca Zordan, Mathew Miller and colleagues from the Johnson laboratory at the University of California, San Francisco, USA.

Previous work on white-opaque switching had identified some of the regulators that are involved - the a $1-\alpha 2$ heterodimer, which is encoded by the mating-type loci, the transcriptional regulator Efg1 and the master regulator Wor 1 - and Zordan and colleagues were interested in completing the regulatory picture. They began by exploiting the differences in gene expression that exist between these different cell types. The authors focused initially on the genes that are upregulated in the opaque state and searched for sequence homology with known fungal transcriptional regulators. Two putative regulators were chosen for further study - Zcf33 and Czf1. Quantitative white-opaque switching assays using $z c f 33 \Delta / z c f 33 \Delta$ and $c z f 1 \Delta / c z f 1 \Delta$ homozygous deletion mutants revealed that the switching frequency of these mutants was greatly reduced compared with that of wild-type cells. Moreover, these mutations could be complemented by the ectopic expression of ZCF33 and $C Z F 1$, which indicates that Zcf33 (which the authors renamed Wor2) and Czf1 are both activators of the opaque state.

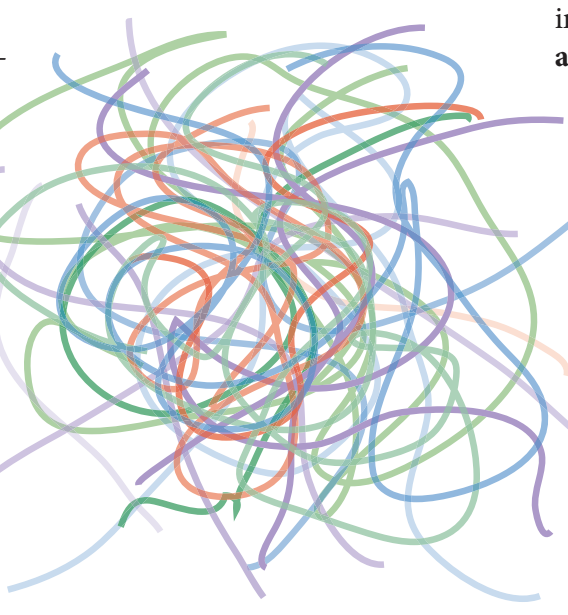

Zordan et al. then began to investigate where these additional transcriptional regulators slot in to the regulatory network that drives white-opaque switching. At first, they looked at the epistatic effects of the ectopic expression of both regulators in different mutant backgrounds. The results revealed that the ectopic expression of WOR1 was sufficient for switching, regardless of whether CZF1 or WOR2 was present, which indicates that CFZ1 and WOR2 both function upstream of WOR1. From previous work, it was known that Worl is needed to maintain the opaque state. In this study, Zordan et al. found that Wor2 is also required to maintain the opaque state, whereas Czf1 is important for white-opaque switching, but not for opaque maintenance. Previous work had also revealed that Efg1, a transcriptional regulator that is upregulated in white cells, could induce switching in some, but not all, $a / \alpha$ strains. To probe this further, the authors constructed $e f g 1 \Delta /$ efg $1 \Delta$ mutants in both a and $\mathbf{a} / \alpha$ backgrounds, and the results suggested that Efg1 functions upstream of the a1- $\alpha 2$-mediated block in switching, and upstream of WOR1.

So, the master regulator Wor1 appears to function downstream of at least three other transcriptional regulators. To investigate whether Wor 1 regulates the transcription of these regulators directly, the authors used 'ChIP on chip' chromatin immunoprecipitation analysis to identify the Wor1-binding sites in the C. albicans genome. These experiments revealed that Wor 1 binds at the intergenic regions upstream of $C Z F 1$, WOR2 and $E F G 1$, and therefore regulates these genes directly.

With this detailed analysis, Zordan, Miller and colleagues have unravelled the interlocking network of positive-feedback loops that controls this epigenetic phenomenon.

Sheilagh Molloy

ORIGINAL RESEARCH PAPER Zordan, R. E. et al. Interlocking transcriptional feedback loops control white-opaque switching in Candida albicans. PLoS Biol. 5, e256 (2007) 